\title{
Notch intracellular domain regulates glioblastoma proliferation through the Notch1 signaling pathway
}

\author{
YIXUAN WANG ${ }^{1,2 *}$, QIAN SUN ${ }^{1,2^{*}}$, RONGXIN GENG ${ }^{1,2}$, HAO LIU $^{1,2}$, FAN'EN YUAN $^{1,2}$, YANG XU $^{1,2}$, \\ YANGZHI QI $^{1,2}$, HONGXIANG JIANG ${ }^{1,2}$, QIANXUE CHEN ${ }^{1,2}$ and BAOHUI LIU ${ }^{1,2}$ \\ ${ }^{1}$ Department of Neurosurgery; ${ }^{2}$ Central Laboratory, Renmin Hospital of Wuhan University, Wuhan, Hubei 430060, P.R. China
}

Received April 9, 2020; Accepted November 24, 2020

DOI: $10.3892 / 01.2021 .12564$

\begin{abstract}
Notch intracellular domain (NICD), also known as the activated form of Notch1 is closely associated with cell differentiation and tumor invasion. However, the role of NICD in glioblastoma (GBM) proliferation and the underlying regulatory mechanism remains unclear. The present study aimed to investigate the expression of NICD and Notch1 downstream gene HES5 in human GBM and normal brain samples and to further detect the effect of NICD on human GBM cell proliferation. For this purpose, western blotting and immunohistochemical staining were performed to analyze the expression of NICD in human GBM tissues, while western blotting and reverse-transcription quantitative PCR experiments were used to analyze the expression of Hes5 in human GBM tissues. A Flag-NICD vector was used to overexpress NICD in U87 cells and compound E and small interfering (si) Notch1 were used to downregulate NICD. Cellular proliferation curves were generated and BrdU assays performed to evaluate the proliferation of U87 cells. The results demonstrated that compared with normal brain tissues, the level of NICD protein in human GBM tissues was upregulated and the protein and mRNA levels of Hes5 were also upregulated in GBM tissues indicating that the Notch1 signaling pathway is activated in GBM. Overexpression of NICD promoted the proliferation of U87 cells in vitro while downregulation of NICD by treatment with compound E or siNotch1 suppressed the proliferation of U87 cells in vitro. In conclusion, NICD was upregulated in human GBM and NICD promoted GBM proliferation via the Notch1 signaling pathway. NICD may be a potential diagnostic marker and therapeutic target for GBM treatment.
\end{abstract}

Correspondence to: Professor Baohui Liu or Professor Qianxue Chen, Department of Neurosurgery, Renmin Hospital of Wuhan University, 238 Jiefang Street, Wuhan, Hubei 430060, P.R. China

E-mail: bliu666@whu.edu.cn

E-mail: chenqx666@whu.edu.cn

${ }^{*}$ Contributed equally

Key words: glioblastoma, proliferation, Hes5, Notch intracellular domain, Notch1

\section{Introduction}

Glioma is a common malignant tumor of the central nervous system and prognosis is extremely poor even after years of treatment (1). Glioblastoma (GBM) as the most malignant type of glioma is classified as grade IV according to the 2016 WHO classification criteria (2). Numerous patients undergo total resection followed by adjuvant chemoradiotherapy, which is the current standard treatment for GBM, but the overall survival and quality of life have been disappointing (3). Currently, GBM has 12,000 newly diagnosed cases annually in the United States (4). The Central Brain Tumor Registry of the United States recently described 1- and 5-year overall survival rates of 40.2 and $5.6 \%$, respectively for GBM from 2000-2015 (5). Abnormal proliferation of cells is an important feature of GBM and inhibiting the proliferation of GBM cells could suppress tumor growth and improve the survival of patients (6). GBM proliferation is associated with a number of molecular pathways, including the neurogenic locus notch homolog protein 1 (Notch1) signaling pathway (7).

The Notch1 signaling pathway participates in cell proliferation, metastasis, embryo development and tissue generation (8). Previous studies have found that the Notch signaling pathway is abnormally activated in gliomas $(9,10)$ and that hyperactivation of Notch1 can promote differentiation and vascularization of malignant glioma stem cells, thereby accelerating tumor invasion and metastasis (11). The most common target genes in the classic notch signaling pathway are members of the HES family of transcription repressors (12). Hairy enhancer of split 1 (Hesl) and hairy enhancer of split 5 (Hes5) are important target genes downstream of the Notch1 signaling pathway, and they have been reported to be associated with GBM proliferation $(13,14)$. Recently, more research revealed that the Notch signaling pathway was closely associated with abnormal proliferation of GBM cells, but the regulatory mechanism behind this remains unclear $(15,16)$. Hence, an improved understanding of Notch pathway components, such as the Notch intracellular domain (NICD) is an important step towards understanding the exact function of Notch and the development of GBM targeted therapy.

In further studies investigating the Notch1 signaling pathway, a molecule called the Notch intracellular domain (NICD) was identified that has attracted the attention of researchers. The intracellular fragment of Notch 1 can be 
cleaved by $\gamma$-secretase to release NICD, the activated form of Notch1 (17). NICD then is transferred into the nucleus where it can act as a transcriptional activation factor (18). The expression of NICD in glioma can be influenced by hypoxia (19). Chronic hypoxia ( $48 \mathrm{~h}$ ) can continuously enhance the expression of hypoxia inducible factor- $2 \alpha$ which can activate the Notch signaling pathway $(20,21)$. The upregulation of NICD induces the activation of Notch1, which promotes GBM invasion and migration by promoting $\beta$-catenin and NF- $\mathrm{KB}$ signaling (22). The role of NICD in the abnormal proliferation of GBM remains unclear.

The aim of the present study was to explore whether the expression of NICD in GBM is different from that in normal brain tissue, and to better understand the role and regulatory mechanism of NICD in GBM. The present study revealed the function of NICD in GBM proliferation and its role in the Notch1 signaling pathway which may provide a new molecular tool for the diagnosis and treatment of GBM.

\section{Materials and methods}

Antibodies and reagents. Primary antibodies used were as follows: i) Anti-NICD [1:1,000 for western blotting and 1:200 for immunohistochemistry (IHC); cat. no. 2421; Cell Signaling Technology, Inc.]; ii) anti-Hes5 (1:1,000 for western blotting; cat. no. sc-13859; Santa Cruz Biotechnology, Inc.); iii) anti-phosphorylated (p)-Histone H3 (1:1,000 for western blotting; cat. no. sc-8656; Santa Cruz Biotechnology, Inc.); iv) anti-Histone H3 (1:1,000 for western blotting; cat. no. GB13102-1; Wuhan Servicebio Technology, Co., Ltd); v) anti-Notch1 (1:2,000 for WB; cat. no. ab27526; Abcam); and vi) anti-GAPDH (1:2,000 for western blotting; cat. no. 5174; Cell Signaling Technology, Inc.). Secondary antibodies used were as follows: i) Alex Fluor 680/790 (1:10,000 for western blotting; cat. no. ANT091; Antgene); ii) HRP-labeled (1:100 for IHC; cat. no. GB23303; Wuhan Servicebio Technology, Co., Ltd). The antibodies used in the BrdU assay were anti-BrdU (1:200; cat. no. 552598; Becton and Dickinson and Company) and Alexa Fluor 594 (1:100; cat. no. A11032; Invitrogen; Thermo Fisher Scientific, Inc.). Compound E (cat. no. AG-CR1-0081; Adipogen Life Sciences, Inc.) was used as an inhibitor of NICD at an intervention concentration of $1 \mu \mathrm{M}$ which was in accordance with the manufacturer's instructions.

Tissue samples. Human control brain tissues and GBM tissues were acquired from the Department of Neurosurgery, Renmin Hospital of Wuhan University (Wuhan, China). GBM tissues were sampled during resection surgeries and stored at $-80^{\circ} \mathrm{C}$. A total of 19 pathologically diagnosed GBM samples were enrolled in this study. All the clinical samples were collected between December 2012 and September 2014. The mean ages were 47 and 35 years for GBM and controls, respectively. The average weight of the sample was generally $\sim 3 \mathrm{~g}$ of which $0.5 \mathrm{~g}$ was used for western blotting and the rest was used for immunohistochemistry and other related experiments. Control brain tissues used in the present study were normal tissues collected from 11 non-tumor patients during emergency surgeries of traumatic brain injury. Patients in the present study had the following inclusion criteria: i) confirmed histological diagnosis of GBM; and ii) performed surgical resection. The exclusion criteria for patients were: i) History of multiple tumors or severe cerebral vascular or cardiovascular diseases (heart stent surgery or admission to the acute ward due to cardiovascular disease within the last 6 months), severe vascular lesions, tuberculosis and extensive acute inflammation (including, but not limited to acute meningitis and vasculitis) within the last 3 months; and ii) participation in a clinical trial within the last 3 months. The histological diagnosis of glioma was confirmed by the pathologists of the Department of Pathology, Renmin Hospital of Wuhan University (Wuhan, China). All tumor samples were subjected to pathological examination and related molecular testing [Methylation of O6-methylguanine-DNA methyltransferase (MGMT)], 1p19q, and isotrate dehydrogenase (IDH)1/IDH2], which were all defined according to the 2016 WHO classification (1). All clinical information for the patients is presented in Table SI. The procurement and use of tissue in the present study were approved by the Institutional Ethics Committee of the Faculty of Medicine, Renmin Hospital of Wuhan University [approval number, 2012LKSZ (010) H]. Written informed consent for use of tissue was obtained from all patients in the present study.

Cell culture. U87 (glioblastoma cell line of unknown origin; CL-0238) was purchased from the Cell Bank of Type Culture Collection of the Chinese Academy of Sciences. The U87 cell line was STR authenticated. Cells were cultured in DMEM high glucose medium (cat. no. GNM12800; Jinuo Biomedical Technology Co., Ltd.) supplemented with $10 \%$ fetal bovine serum (FBS) (cat. no. 04-001-1A; Biological Industries) and $100 \mathrm{U} / \mathrm{ml}$ penicillin and $100 \mu \mathrm{g} / \mathrm{ml}$ streptomycin. Cells were incubated in humidified air with $5 \% \mathrm{CO}_{2}$ at $37^{\circ} \mathrm{C}$.

Plasmid construction and transfection. A Flag-NICD and Flag-control plasmid was provided by Dr Chundong $\mathrm{Yu}$ (Xiamen University, Xiamen, China) and its construction has been described in a previous study by Lin et al (23). An siRNA (siNotch1) and a non-targeting control siRNA (siCtrl) were purchase from Thermo Scientific Inc. The sequences used were as follows: siRNA-Notch1, sense 5'-GCAACCUGCAGUGUA AUAATT-3' and antisense 5'-UUAUUACACUGCAGGUUG CTT-3'; siRNA-non-targeting control, sense 5'-UUCUCCGAA CGUGUCACGUTT-3' and antisense 5'-ACGUGACACGUU CGGAGAATT-3'. U87 cells were seeded in 6-well plates at a density of about $5 \times 10^{5}$ cells $/$ well and cultured overnight at $37^{\circ} \mathrm{C}$. Plasmids $2 \mu \mathrm{g}$ per well were transfected by Lipofectamine $3000^{\circledR}$ transfection reagent (Thermo Fisher Scientific, Inc.), and siRNA $200 \mathrm{pM}$ per well was transfected by Lipofectamine RNAiMax reagent (Thermo Fisher Scientific, Inc.) according to the manufacturer's instructions. Plasmids and siRNA were incubated with Lipofectamine $3000^{\circledR}$ transfection reagent for $15 \mathrm{~min}$ at room temperature before transfection and U87 cells were transfected for $48 \mathrm{~h}$ prior to performing subsequent experiments.

Western blotting. U87 cell samples and brain tissues were lysed on ice in RIPA buffer (50 mm Tris, $150 \mathrm{~mm} \mathrm{NaCl}, 0.5 \%$ EDTA and $0.5 \%$ NP-40) with PMSF protease inhibitors (Beyotime Institute of Biotechnology) and an inhibitor cocktail (Roche Diagnostics). The concentration of total proteins was detected 
using a bicinchoninic acid (BCA) kit (Biosharp Life Sciences). Protein was loaded and separated by $10 \%$ SDS-PAGE and then transferred onto PVDF membranes (cat. no. 88520; Thermo Fisher Scientific, Inc.). In total $\sim 20 \mu \mathrm{g} /$ well of protein was added. After blocking with 5\% skimmed milk for $30 \mathrm{~min}$ at room temperature, membranes were incubated with primary antibodies overnight and with secondary antibodies for $1 \mathrm{~h}$. The samples were visualized with a LI-COR Odyssey Infrared Imaging System (LI-COR Biosciences). GAPDH was used as a loading control.

Immunohistochemical staining. Human brain tissues were fixed in $4 \%$ paraformaldehyde at room temperature for $48 \mathrm{~h}$ and embedded in paraffin and cut into sections. The thickness of sections used for immunohistochemical staining was $6-\mu \mathrm{m}$ and these sections were cut using a paraffin section machine (cat. no. HM340E; Thermo Fisher Scientific, Inc.). After paraffin sections were deparaffinized at $65^{\circ} \mathrm{C}$ for $2 \mathrm{~h}$, hydrated with 100/95/75\% ethanol for $10 \mathrm{~min}$ each, antigen recovery was performed by exposing sections to $0.01 \mathrm{~mol} / \mathrm{l}$ citrate buffer (pH 6.0) at $100^{\circ} \mathrm{C}$ for $20 \mathrm{~min}$. Endogenous peroxidase was removed by treatment with $3 \% \mathrm{H}_{2} \mathrm{O}_{2}$. Samples were blocked with $1 \%$ BSA (Amresco, LLC) at room temperature for $1 \mathrm{~h}$ and were then incubated with primary antibodies at $37^{\circ} \mathrm{C}$ overnight. Following 3 washes with PBS, the sections were incubated with secondary antibody for $1 \mathrm{~h}$ at room temperature. DAB (Wuhan Servicebio Technology, Co., Ltd.) was used for dyeing and the nuclei were stained by hematoxylin at room temperature for $30 \mathrm{sec}$. Images were captured with an Olympus BX51 fluorescence microscope (Olympus Corporation).

Reverse-transcription quantitative (RT-q) PCR. Brain tissues were ground with a Hybrid Mill (cat. no. MM400; RETSCH $\mathrm{GmbH}$ ) for RNA extraction. RNA was isolated using an RNA isolation kit (Takara Bio, Inc.) and cDNA was synthesized from $1 \mu \mathrm{g}$ of total RNA using a PrimeScript ${ }^{\mathrm{TM}} \mathrm{RT}$ reagent kit (Takara Bio, Inc.) at $37^{\circ} \mathrm{C}$ for $5 \mathrm{~min}, 42^{\circ} \mathrm{C}$ for $30 \mathrm{~min}$ and then $85^{\circ} \mathrm{C}$ for $5 \mathrm{sec}$. RT-qPCR experiments were performed using a SYBR ${ }^{\circledR}$ Premix Ex Taq ${ }^{\mathrm{TM}} \mathrm{Kit}$ (Takara Bio, Inc.) in a CFX Connect system (Bio-Rad Laboratories, Inc.). The RT-PCR thermocycling conditions were as follows: $95^{\circ} \mathrm{C}$ for $30 \mathrm{sec}$ for initial denaturation, $95^{\circ} \mathrm{C}$ for $5 \mathrm{sec}$ followed by 40 cycles of $60^{\circ} \mathrm{C}$ for $40 \mathrm{sec}$ for PCR reaction stage and the dissociation was set by CFX Connect system by default. Gene expression levels were determined by the $2^{-\Delta \Delta \mathrm{Cq}}$ threshold cycle method (24). The forward and reverse PCR primer sequences were as follows: Hes5, forward, 5'GGAATTCCAATGGCCCCCAGC ACTGTG-3' and reverse, 5'-GGGTACCCCACGGCCACA GTGCTGG-3' and GAPDH, forward, 5'-GAGTCAACGGAT TTGGTCGT-3' and reverse, 5'-TTGATTTTGGAGGGATCT CG-3'. Normalizations across samples were performed using the geometric average of the constitutive gene expression of GAPDH.

BrdU assay. Cell samples were treated with BrdU reagent (cat. no. 00-0103; Invitrogen; Thermo Fisher Scientific, Inc.) for $30 \mathrm{~min}$ at $37^{\circ} \mathrm{C}$. Prior to harvesting the cells, $200 \mu \mathrm{l}$ of $1 \mathrm{X} \mathrm{BrdU/well} \mathrm{was} \mathrm{added.} \mathrm{Next,} \mathrm{the} \mathrm{cells} \mathrm{were} \mathrm{fixed} \mathrm{in}$ $4 \%$ paraformaldehyde for $10 \mathrm{~min}$ at room temperature, lysed by treatment with Triton X-100 for $5 \mathrm{~min}$ at room temperature and
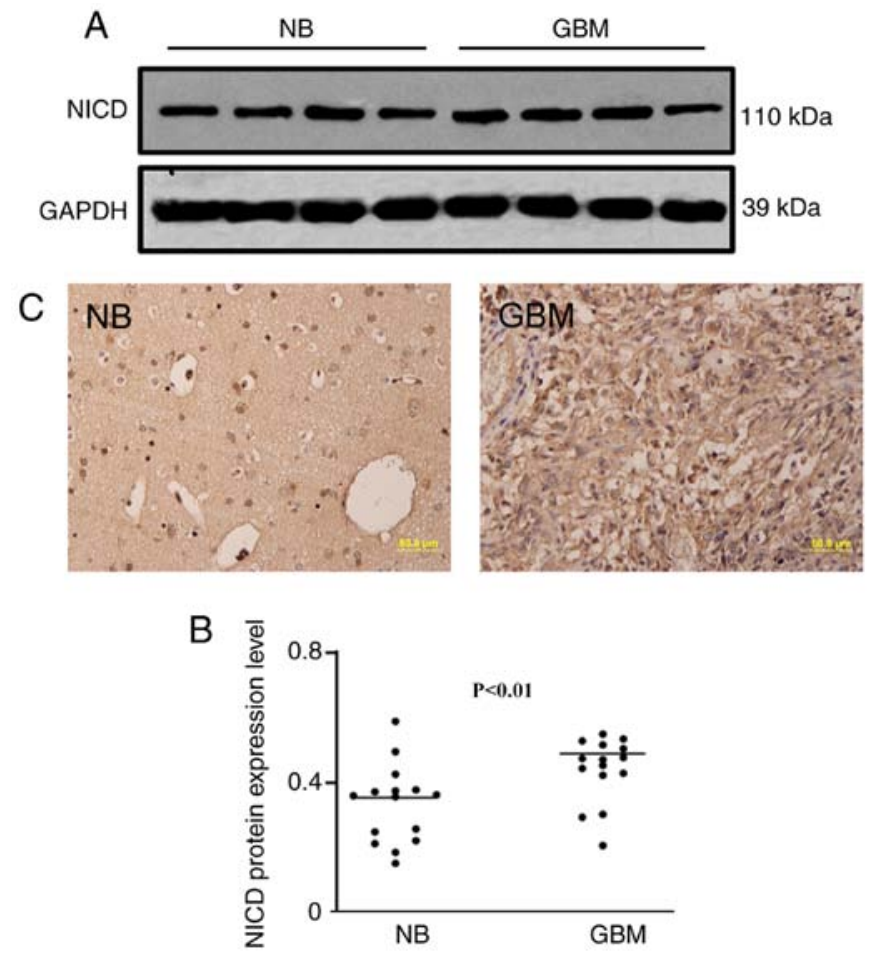

Figure 1. NICD is upregulated in human GBM tissues. (A and B) Western blotting demonstrated that the NICD protein level was significantly increased in human GBM tissue compared with normal brain tissue $(\mathrm{P}<0.01)$ (C) Immunohistochemical staining demonstrated that the expression level of NICD was increased in GBM compared with normal brain tissue. Scale bar, $50 \mu \mathrm{m}$. NB, normal brain; GBM, glioblastoma; NICD, Notch intracellular domain.

blocked in BSA for $1 \mathrm{~h}$ at room temperature. The sample was incubated with a primary antibody (anti-BrdU) at $4^{\circ} \mathrm{C}$ overnight and a secondary antibody (Alexa Fluor 594) at room temperature for $1 \mathrm{~h}$. Nuclei were stained with diamidino-phenyl-indole (DAPI) (cat. no. ANT046; Wuhan Antejie Biotechnology Co., Ltd.). Fluorescence images of BrdU were visualized with an Olympus BX51 microscope (Olympus Corporation), which is a confocal microscope. The number of BrdU-positive cells was quantified using ImageJ software (v.1.48; National Institutes of Health).

Statistical analysis. Statistical analyses performed using SPSS 20.0 software (IBM Corp.). The data are presented as the mean \pm standard deviation of three independent repeats. Differences between two groups were analyzed using an unpaired Student's t-test. $\mathrm{P}<0.05$ was considered to indicate a statistically significant difference.

\section{Results}

NICD is upregulated in human GBM tissues compared with normal brain tissues. First, to explore the clinical value of NICD, its expression level was detected in 11 human normal brain samples and 19 human GBM samples. Western blotting demonstrated that the NICD protein level was significantly increased in human GBM tissues compared with normal brain tissues (Fig. 1A and B). Immunohistochemical staining also demonstrated an increased expression level of NICD in GBM 
A

A

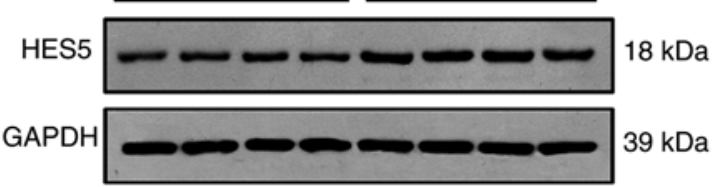

B

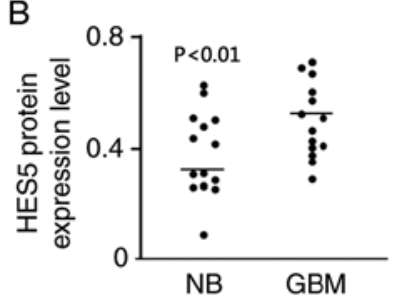

C

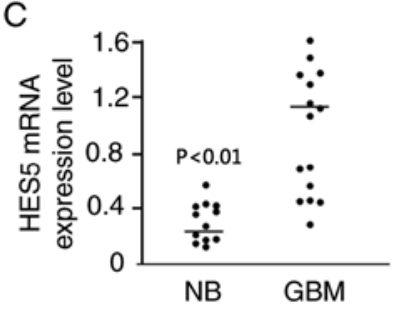

Figure 2. Notch1 signaling pathway is activated in GBM tissues. (A and B) Western blotting demonstrated that the Hes5 protein level was significantly increased in human GBM tissue compared with normal brain tissue $(\mathrm{P}<0.01)$. (C) Reverse-transcription qPCR demonstrated that Hes5 mRNA was significantly increased in human GBM tissue compared with normal brain tissue $(\mathrm{P}<0.01)$. NB, normal brain; GBM, glioblastoma; NICD, Notch intracellular domain.
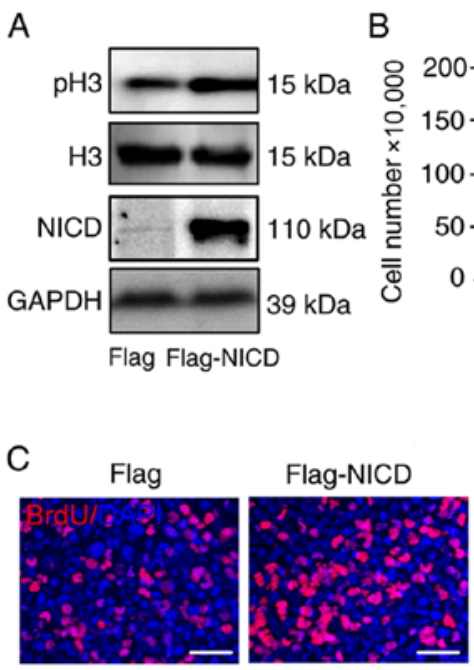

B
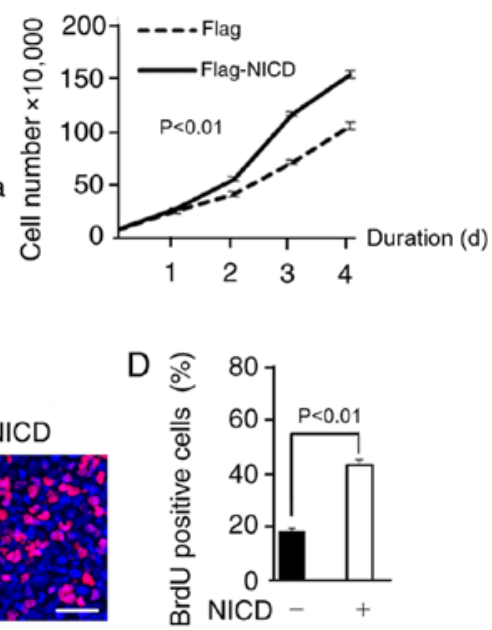

Figure 3. NICD promotes the proliferation of glioblastoma cells. (A) NICD was upregulated by the transfection of Flag-NICD plasmid and overexpressed NICD increased the protein level of pH3. (B) Cell growth was promoted in NICD group compared with control group as can be seen from the proliferation curve $(\mathrm{P}<0.01)$, and the data of each time point in panel $\mathrm{B}$ represent the average of three measurements $(\mathrm{P} 1=0.0082 ; \mathrm{P} 2<0.0001 ; \mathrm{P} 3<0.0001$; and $\mathrm{P} 4=0.0003$. p1, p2, p3, p4 represent the p-value of the time points of the first, second, third and fourth day, respectively). (C and D) BrdU assay demonstrated that the proportion of BrdU-positive cells was increased in NICD group compared with control group $(\mathrm{P}<0.01)$. Scale bar, $50 \mu \mathrm{m}$. NICD, Notch intracellular domain; $\mathrm{pH} 3$, phosphohistone-H3.

compared with normal brain tissue (Fig. 1C). Both results demonstrated an obvious increase in expression of NICD in human GBM compared with healthy brain tissue.

The Notchl signaling pathway is activated in GBM tissues. To investigate the activation of the Notch1 signaling pathway in GBM, the expression of Hes5 was investigated as it is an important target gene of Notch1 signaling (25). The findings demonstrated that Hes 5 protein levels were significantly
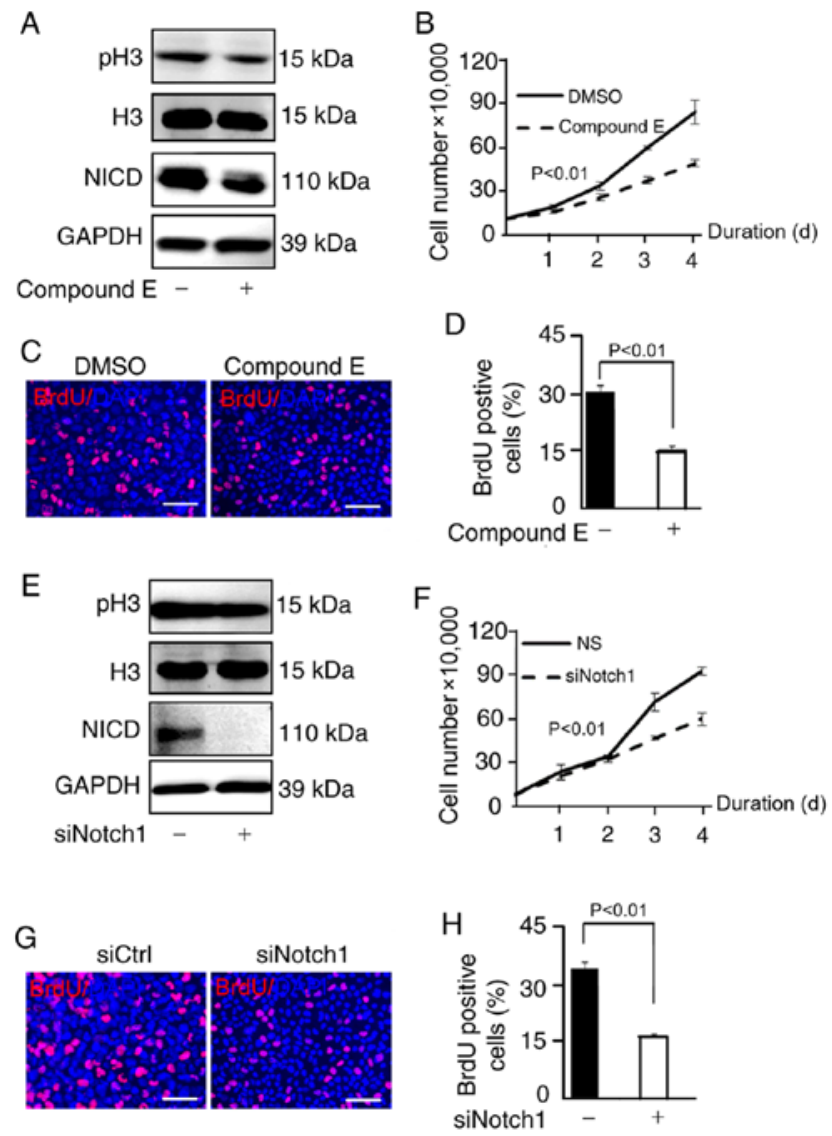

Figure 4. Downregulation of NICD suppresses the proliferation of glioblastoma cells. (A) NICD was downregulated by the use of compound E and decreased NICD suppressed the protein level of pH3 in U87 cells. (B) U87 cell growth was suppressed in the compound $\mathrm{E}$ group compared with the DMSO group as shown in the proliferation curve $(\mathrm{P}<0.01)$. ( $\mathrm{C}$ and $\mathrm{D})$ BrdU assay demonstrated that the proportion of BrdU-positive U87 cells was decreased in compound $\mathrm{E}$ group compared with DMSO group $(\mathrm{P}<0.01)$. (E) NICD was downregulated by the intervention of siNotch1 and decreased NICD suppressed the protein level of pH3 in U87 cells. (F) U87 cell growth was suppressed in siNotch1 group compared with siCtrl group as shown in the proliferation curve $(\mathrm{P}<0.01)$. $(\mathrm{G}$ and $\mathrm{H})$ BrdU assay demonstrated that the proportion of BrdU-positive U87 cells was decreased in siNotch1 group compared with siCtrl group $(\mathrm{P}<0.01)$. Scale bar, $50 \mu \mathrm{m}$. NICD, Notch intracellular domain; $\mathrm{pH} 3$, phosphohistone-H3; si, small interfering.

upregulated in human GBM tissues compared with normal brain tissues (Fig. 2A and B). In addition, Hes5 mRNA expression was also increased in human GBM tissues compared with normal brain tissues (Fig. 2C). Both results indicated that the Notch1 signaling pathway was activated in human GBM.

NICD promotes the proliferation of U87 cells. NICD protein was overexpressed by transfection of U87 cells with a Flag-NICD plasmid (Fig. 3A) and the change in cellular proliferation induced by NICD was assessed. The level of histone H3 phosphorylation reflects cell proliferation (26). An increase in the phosphorylation level of histone $\mathrm{H} 3$ in the NICD group compared to the control group was observed by western blotting (Fig. 3A). Compared with the control group, the group overexpressing NICD exhibited a significant increase in the number of U87 cells as seen in the proliferation curve (Fig. 3B), and BrdU assays demonstrated that the proportion of BrdU-positive U87 cells increased (Fig. 3C and D). These 
data demonstrated that NICD induced the proliferation of U87 cells in vitro.

Downregulation of NICD suppresses the proliferation of U87 cells. To further confirm the relationship between NICD and U87 cell proliferation, U87 cells were observed when NICD was downregulated. Compound $\mathrm{E}$ is an inhibitor of $\gamma$-secretase and thus has an inhibitory effect on NICD (27). The expression of NICD and the level of $\mathrm{H} 3$ phosphorylation was clearly downregulated after cells were treated with compound $\mathrm{E}$ (Fig. 4A). Consequently, the number of U87 cells was significantly decreased compared with the control group (DMSO) as shown in the proliferation curve (Fig. 4B), and the proportion of BrdU-positive U87 cells was significantly reduced compared with the control group (DMSO) in the BrdU assay (Fig. 4C and D). In addition, Brdu postive cells in the control group (NICD-) (Fig. 3D) were less compared with the control group (compound E-) (Fig. 4D) as vector transfection in the NICD- group had a certain inhibiting effect on cells while $3 \mu \mathrm{m}$ DMSO treatment in the compound E- group had almost no toxic effect. In addition, w U87 cells were transfected with siNotch1 to suppress the activity of the Notch1 signaling pathway; the results demonstrated that the NICD level and the level of $\mathrm{H} 3$ phosphorylation were reduced compared with the control group (siNotch1-) (Fig. 4E) and the proliferation of U87 cells was inhibited as well compared with the control group (siCtrl) (Fig. 4F-H). In conclusion, NICD was inhibited by compound $\mathrm{E}$ and knockdown of Notch1 also reduced the expression of NICD and suppressed the proliferation of U87 cells.

\section{Discussion}

The Notch1 signaling pathway has been reported to associate with various cancers, such as lung cancer and gastric cancer and participate in several neoplastic biological behaviors including drug resistance and apoptosis $(28,29)$. Although some studies have demonstrated that Notch1 is related to the abnormal proliferation of GBM cells (30-33), the specific regulatory mechanism of this remains unclear. As significant effectors downstream of the Notch1 signaling pathway, Hes1 and Hes5 have recently received increasing attention (25). Hes1 is expressed at low levels in the human brain, and several studies have detected uninduced Hes1 expression in GBM tissues $(34,35)$. In comparison, the Hes5 expression level was relatively high (34), however, the expression of Hes5 in human GBM is still controversial and remains to be solved. Hence, the present study investigated the level of Hes5 fixed GBM samples. Interestingly, the expression of Hes5 was higher at both the protein and mRNA levels in the GBM samples compared with normal control brain samples, indicating an activated state of the Notch1 signaling pathway in GBM tissues which has not been previously reported to the best of our knowledge. According to the existing literature, HES5 serves an important role in the regulation of mammalian neuronal differentiation and the maintenance of neural stem cells and can actively regulate the self-renewal of neuronal stem cells (13). Based on the findings of the present study, it may be speculated that HES5 in GBM may have a significant impact on the occurrence and development of GBM.
NICD is the activated form of Notch1 and it serves an important role in the Notch1 signaling pathway (36). Notably, most studies of NICD explored is effect on cell differentiation (19), invasion (22) and apoptosis in glioma (37). It is generally accepted that the growth, metastasis, and invasion of tumors are inseparable from the proliferation of tumor cells (38), however, it was still unclear whether NICD could regulate cell proliferation in GBM. Hence, the present study explored the connection between NICD and proliferation to fill in the gaps in the knowledge regarding GBM.

In the present study, the expression of NICD in human GBM tissues which has not been thoroughly explored in previous research was explored and the findings demonstrated an increase in NICD protein level in GBM tissues compared with normal brain tissues. To determine whether NICD could regulate cell proliferation in GBM, NICD was overexpressed in vitro using the plasmid Flag-NICD. Phosphorylated histone H3 levels, a key marker of cell proliferation have been demonstrated to be upregulated by induced cell growth (39). The findings of the present study showed demonstrated that phosphorylated histone $\mathrm{H} 3$ levels were increased and cell growth was promoted following the overexpression of NICD. In conclusion, in the present study NICD was activated in GBM tissues and promoted GBM proliferation in vitro.

In addition, to explore whether NICD regulates cell proliferation through the Notch1 signaling pathway, compound $\mathrm{E}$ and siNotch1 were used to suppress the activation of NICD and Notch1, respectively. Compound E, a selective inhibitor of $\gamma$-secretase that has the ability to suppress the expression of NICD (40) was observed to decrease NICD protein levels and phosphorylated histone $\mathrm{H} 3$ levels as well as to repress the growth of U87 cells in the present study. In addition, it has been reported that overexpression of NICD in PC12 cells can promote cell apoptosis and inhibit cell proliferation by regulating $S$ phase arrest (41). Similar results were obtained in the present study where NICD was inhibited and cell proliferation suppressed when Notch1 was knocked down by siNotch 1 . Hence, in the present study a reduced level of NICD suppressed the proliferation of GBM cells and NICD regulated proliferation through the Notch1 signaling pathway.

GBM is still the most malignant type of glioma, and it exhibits abnormal proliferation and a high mortality rate according to the statistical analysis of glioma cases in US between 2010 and 2014 (4). Early diagnosis and precision treatments have great significance for providing an improved prognosis for patients with GBM (42). The findings of the present study emphasized the significance of NICD in the Notch1 signaling pathway regulation of the proliferation of GBM. Hence, NICD may be a potential diagnostic marker and therapeutic target for GBM.

\section{Acknowledgements}

Not applicable.

\section{Funding}

No funding was received. 


\section{Availability of data and materials}

The datasets used and/or analyzed during the current study are available from the corresponding author on reasonable request.

\section{Authors' contributions}

BL and QC designed the research. YW and QS performed the experiments. RG, HL, FY and YX analyzed the data and wrote the manuscript. YQ and $\mathrm{HJ}$ participated in data analysis and revised the article for important intellectual content. All authors have read and approved the final manuscript.

\section{Ethics approval and consent to participate}

All tissue specimens used in this study were approved by the Institutional Ethics Committee of the Faculty of Medicine, Renmin Hospital of Wuhan University (approval no. 2012LKSZ (010) H) (Wuhan, China). Written informed consent was obtained from all the patients.

\section{Patient consent for publication}

Not applicable.

\section{Competing interests}

The authors declare that they have no competing interests.

\section{References}

1. Reni M, Mazza E, Zanon S, Gatta G and Vecht CJ: Central nervous system gliomas. Crit Rev Oncol Hematol 113: 213-234, 2017.

2. Louis DN, Perry A, Reifenberger G, von Deimling A, Figarella-Branger D, Cavenee WK, Ohgaki H, Wiestler OD, Kleihues P and Ellison DW: The 2016 World Health Organization classification of tumors of the central nervous system: A summary. Acta Neuropathol 131: 803-820, 2016.

3. Le Rhun E, Rhun EL, Taillibert S and Chamberlain MC: The future of high-grade glioma: Where we are and where are we going. Surg Neurol Int 6 (Suppl 1): S9-S44, 2015.

4. Ostrom QT, Gittleman H, Liao P, Vecchione-Koval T, Wolinsky Y, Kruchko C and Barnholtz-Sloan JS: CBTRUS Statistical Report: Primary brain and other central nervous system tumors diagnosed in the United States in 2010-2014. Neuro Oncol 19 (suppl_5): v1-v88, 2017.

5. Ostrom QT, Gittleman H, Truitt G, Boscia A, Kruchko C and Barnholtz-Sloan JS: CBTRUS statistical report: Primary brain and other central nervous system tumors diagnosed in the United States in 2011-2015. Neuro Oncol 20 (suppl_4): iv1-iv86, 2018.

6. Han D, Yu T, Dong N, Wang B, Sun F and Jiang D: Napabucasin, a novel STAT3 inhibitor suppresses proliferation, invasion and stemness of glioblastoma cells. J Exp Clin Cancer Res 38: 289, 2019.

7. Ji Y, Sun Q, Zhang J and Hu H: MiR-615 inhibits cell proliferation, migration and invasion by targeting EGFR in human glioblastoma. Biochem Biophys Res Commun 499: 719-726, 2018.

8. Gil-García B and Baladrón V: The complex role of NOTCH receptors and their ligands in the development of hepatoblastoma, cholangiocarcinoma and hepatocellular carcinoma. Biol Cell 108: 29-40, 2016.

9. Stockhausen MT, Kristoffersen K and Poulsen HS: The functional role of Notch signaling in human gliomas. Neuro Oncol 12 : 199-211, 2010

10. Lino MM, Merlo A and Boulay JL: Notch signaling in glioblastoma: A developmental drug target? BMC Med 8: 72, 2010.
11. Guichet PO, Guelfi S, Teigell M, Hoppe L, Bakalara N, Bauchet L, Duffau H, Lamszus K, Rothhut B and Hugnot JP: Notch1 stimulation induces a vascularization switch with pericyte-like cell differentiation of glioblastoma stem cells. Stem Cells 33: 21-34, 2015.

12. Aster JC, Pear WS and Blacklow SC: The varied roles of Notch in cancer. Annu Rev Pathol 12: 245-275, 2017.

13. Ohtsuka T, Ishibashi M, Gradwohl G, Nakanishi S, Guillemot F and Kageyama R: Hes1 and Hes5 as notch effectors in mammalian neuronal differentiation. EMBO J 18: 2196-2207, 1999.

14. Zhang YM, Chen SX, Dai QF, Jiang ST, Chen AL, Tang CZ and Zhang YQ: Effect of acupuncture on the notch signaling pathway in rats with brain injury. Chin J Integr Med 24: 537-544, 2018.

15. Purow BW, Haque RM, Noel MW, Su Q, Burdick MJ, Lee J, Sundaresan T, Pastorino S, Park JK, Mikolaenko I, et al: Expression of Notch-1 and its ligands, Delta-like-1 and Jagged-1, is critical for glioma cell survival and proliferation. Cancer Res 65: 2353-2363, 2005.

16. Hu GW, Wu L, Kuang W, Chen Y, Zhu XG, Guo H and Lang HL: Knockdown of linc-OIP5 inhibits proliferation and migration of glioma cells through down-regulation of YAP-NOTCH signaling pathway. Gene 610: 24-31, 2017.

17. Hu S, Chen Q, Lin T, Hong W, Wu W, Wu M, Du X and Jin R: The function of Notch1 intracellular domain in the differentiation of gastric cancer. Oncol Lett 15: 6171-6178, 2018.

18. Tamura K, Taniguchi Y, Minoguchi S, Sakai T, Tun T, Furukawa T and Honjo T: Physical interaction between a novel domain of the receptor Notch and the transcription factor RBP-J kappa/Su(H). Curr Biol 5: 1416-1423, 1995.

19. Hu YY, Fu LA, Li SZ, Chen Y, Li JC, Han J, Liang L, Li L, Ji CC, Zheng MH and Han H: Hif- $1 \alpha$ and Hif- $2 \alpha$ differentially regulate Notch signaling through competitive interaction with the intracellular domain of Notch receptors in glioma stem cells. Cancer Lett 349: 67-76, 2014

20. Renfrow JJ, Soike MH, Debinski W, Ramkissoon SH, Mott RT, Frenkel MB, Sarkaria JN, Lesser GJ and Strowd RE: Hypoxia-inducible factor $2 \alpha$ : A novel target in gliomas. Future Med Chem 10: 2227-2236, 2018.

21. Patel SA and Simon MC: Biology of hypoxia-inducible factor-2alpha in development and disease. Cell Death Differ 15: 628-634, 2008.

22. Zhang X, Chen T, Zhang J, Mao Q, Li S, Xiong W, Qiu Y, Xie Q and Ge J: Notch1 promotes glioma cell migration and invasion by stimulating $\beta$-catenin and NF- $\kappa \mathrm{B}$ signaling via AKT activation. Cancer Sci 103: 181-190, 2012.

23. Lin X, Liu B, Yang X, Yue X, Diao L, Wang J and Chang J: Genetic deletion of Rnd 3 results in aqueductal stenosis leading to hydrocephalus through up-regulation of Notch signaling. Proc Natl Acad Sci USA 110: 8236-8241, 2013.

24. Livak KJ and Schmittgen TD: Analysis of relative gene expression data using real-time quantitative PCR and the 2(-Delta Delta C(T)) method. Methods 25: 402-408, 2001.

25. Ronchi CL, Sbiera S, Altieri B, Steinhauer S, Wild V, Bekteshi M, Kroiss M, Fassnacht M and Allolio B: Notch1 pathway in adrenocortical carcinomas: Correlations with clinical outcome. Endocr Relat Cancer 22: 531-543, 2015.

26. Elmaci İ, Altinoz MA, Sari R and Bolukbasi FH: Phosphorylated histone $\mathrm{H} 3$ (PHH3) as a novel cell proliferation marker and prognosticator for meningeal tumors: A short review. Appl Immunohistochem Mol Morphol 26: 627-631, 2018.

27. Yang T, Arslanova D, Gu Y, Augelli-Szafran C and Xia W: Quantification of gamma-secretase modulation differentiates inhibitor compound selectivity between two substrates Notch and amyloid precursor protein. Mol Brain 1: 15, 2008.

28. Jun HT, Stevens J and Kaplan-Lefko P: Top NOTCH targets: Notch signaling in cancer. Drug Development Research 69: 319-328, 2010.

29. Pancewicz-Wojtkiewicz J: Epidermal growth factor receptor and notch signaling in non-small-cell lung cancer. Cancer Med 5: 3572-3578, 2016

30. Feng HB, Wang J, Jiang HR, Mei X, Zhao YY, Chen FR, Qu Y, Sai K, Guo CC, Yang QY, et al: $\beta$-elemene selectively inhibits the proliferation of glioma stem-like cells through the downregulation of Notch1. Stem Cells Transl Med 6: 830-839, 2017.

31. Wang BQ, Yang B, Yang HC, Wang JY, Hu S, Gao YS and Bu XY: MicroRNA-499a decelerates glioma cell proliferation while accelerating apoptosis through the suppression of Notch1 and the MAPK signaling pathway. Brain Res Bull 142: 96-106, 2018. 
32. Hai L, Zhang C, Li T, Zhou X, Liu B, Li S, Zhu M, Lin Y, Yu S, Zhang K, et al: Notch1 is a prognostic factor that is distinctly activated in the classical and proneural subtype of glioblastoma and that promotes glioma cell survival via the NF- $\kappa \mathrm{B}(\mathrm{p} 65)$ pathway. Cell Death Dis 9: 158, 2018.

33. Liu Q, Zheng JM, Chen JK, Yan XL, Chen HM, Nong WX and Huang HQ: Histone deacetylase 5 promotes the proliferation of glioma cells by upregulation of Notch 1 . Mol Med Rep 10 2045-2050, 2014.

34. Cheung HC, Corley LJ, Fuller GN, McCutcheon IE and Cote GJ: Polypyrimidine tract binding protein and Notch1 are independently re-expressed in glioma. Mod Pathol 19: 1034-1041, 2006.

35. Saito N, Fu J, Zheng S, Yao J, Wang S, Liu DD, Yuan Y, Sulman EP, Lang FF, Colman H, et al: A high Notch pathway activation predicts response to $\gamma$ secretase inhibitors in proneural subtype of glioma tumor-initiating cells. Stem Cells 32: 301-312, 2014.

36. Liu B, Lin X, Yang X, Dong H, Yue X, Andrade KC, Guo Z, Yang J, Wu L, Zhu X, et al: Downregulation of RND3/RhoE in glioblastoma patients promotes tumorigenesis through augmentation of notch transcriptional complex activity. Cancer Med 4: 1404-1416, 2015.

37. Wang Y, Wang H, Ge $\mathrm{H}$ and Yang Z: AG-1031 induced autophagic cell death and apoptosis in C6 glioma cells associated with Notch-1 signaling pathway. J Cell Biochem 119: 5893-5903, 2018.
38. Xie Q, Mittal S and Berens ME: Targeting adaptive glioblastoma: An overview of proliferation and invasion. Neuro Oncol 16 $1575-1584,2014$

39. Villani V, Mahadevan KK, Ligorio M, Fernández-Del Castillo C, Ting DT, Sabbatino F, Zhang I, Vangel M, Ferrone S, Warshaw AL, et al: Phosphorylated histone H3 (PHH3) is a superior proliferation marker for prognosis of pancreatic neuroendocrine tumors. Ann Surg Oncol 23: 609-617, 2016.

40. Arumugam TV, Cheng YL, Choi Y, Choi YH, Yang S, Yun YK, Park JS, Yang DK, Thundyil J, Gelderblom M, et al: Evidence that gamma-secretase-mediated Notch signaling induces neuronal cell death via the nuclear factor-kappaB-Bcl-2-interacting mediator of cell death pathway in ischemic stroke. Mol Pharmacol 80: 23-31, 2011.

41. Li B, Duan P, Han X, Yan W and Xing Y: NICD inhibits cell proliferation and promotes apoptosis and autophagy in PC12 cells. Mol Med Rep 16: 2755-2760, 2017.

42. Alexander BM and Cloughesy TF: Adult glioblastoma. J Clin Oncol 35: 2402-2409, 2017.

(i) $(-)$ This work is licensed under a Creative Commons Attribution-NonCommercial-NoDerivatives 4.0 International (CC BY-NC-ND 4.0) License. 